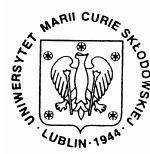

Annales UMCS Informatica AI 8(1) (2008) 133-149

10.2478/v10065-008-0013-5

Annales UMCS

Informatica

Lublin-Polonia

Sectio AI

http://www.annales.umcs.lublin.pl/

\title{
Controllability of second order infinite dimensional dynamical systems with delays
}

\author{
Jerzy Respondek ${ }^{*}$ \\ Department of Theory of Information, Institute of Computer Science, Silesian Technical \\ University, Faculty of Automatic Control, Electronics and Computer Science, \\ Akademicka 16, 44-100 Gliwice, Poland
}

\begin{abstract}
The article is devoted to analysing the approximate absolute and approximate relative controllability of a given type second order infinite dimensional system. The considered dynamical system is governed by the evolution equation with three damping terms and three terms without derivatives. Following this aim, spectral theory for linear unbounded operators is involved. At first the representation of considered infinite dimensional dynamical system by the infinite series of finite dimensional systems is given. Next, two theorems on necessary and sufficient conditions of approximate absolute and approximate relative controllability of the considered system are formulated and proved. Finally, proven theorems are applied to the analysis of the elastic beam.
\end{abstract}

\section{Problem statement}

Let us consider the dynamical system described by the following abstract differential equation:

$$
\begin{aligned}
& \frac{d^{2} x(t)}{d t^{2}}+2\left(\alpha_{0}+\alpha_{1} A+\alpha_{2} A^{\frac{1}{2}}\right) \frac{d x(t)}{d t}+\left(\beta_{0}+\beta_{1} A+\beta_{2} A^{\frac{1}{2}}\right) x(t) \\
& =\sum_{k=0}^{M} B_{k} u\left(t-h_{k}\right), \quad t>0
\end{aligned}
$$

with the initial conditions:

$$
x(0)=x_{0} \in D(A), \dot{x}(0)=x_{1} \in X,
$$

where $x(t) \in X$ ( $X$ is a Hilbert space) and $\alpha_{i} \geq 0, \beta_{i} \geq 0, i=0,1,2$ are the real constant coefficients, $B_{k}$ are $n \times p$ dimensional constant matrices. The constant delays $h_{k}$ fulfils:

\footnotetext{
*e-mail address: Jerzy.Respondek@polsl.pl
} 


$$
0=h_{0}<h_{1}<\ldots<h_{k}<\ldots<h_{M} .
$$

The controls $u \in L_{\text {loc }}^{2}\left(\left[t_{0}, \infty\right), R^{p}\right)$. Furthermore, it is assumed that $A: X \supset D(A) \rightarrow X$ is a linear, generally unbounded, selfadjoint and positivedefinite operator with domain $\mathrm{D}(A)$ dense in $X$ and compact resolvent $R(\lambda, A)$ for all $\lambda$ in the resolvent set $\rho(A)$ (assumption 1).

The physical interpretation of equation (1) encompasses a broad class of real systems in this form and depends on a particular form of the $A$ operator and of the coefficients $\alpha_{i}$ and $\beta_{i}, i=0,1,2$.

It is well known that the operator $A$ has the following spectral properties [1-4]:

- Operator $A$ has only a purely discrete point spectrum consisting entirely of distinct real positive eigenvalues $\lambda_{i}$ each with finite multiplicity $m_{i}$ $\left(m_{i}<\infty\right)$ :

$$
0<\lambda_{1}<\lambda_{2}<\ldots<\lambda_{i}<\lambda_{i+1}<\ldots, \quad \lim _{i \rightarrow \infty} \lambda_{i}=\infty
$$

- The eigenfunctions of operator $A\left\{\phi_{i j}, i=1,2,3, \ldots, j=1,2, \ldots, m_{i}\right\}$ form complete orthogonal system in Hilbert space $X$ and after ortogonalization form complete orthonormal system in Hilbert space $X$. Hence, for every $x \in X$ the following unique expansion holds true:

$$
x=\sum_{i=1}^{\infty} \sum_{j=1}^{m_{i}}<x, \phi_{i j}>_{X} \phi_{i j},
$$

- Operator $A$ has the following spectral resolution:

$$
\begin{gathered}
\underset{x \in D(A)}{\forall} A x=\sum_{i=1}^{\infty} \sum_{j=1}^{m_{i}} \lambda_{i}<x, \phi_{i j}>_{X} \phi_{i j}, \\
D(A)=\left\{x \in X: \sum_{i=1}^{\infty} \sum_{j=1}^{m_{i}} \lambda_{i}^{2}\left|<x, \phi_{i j}>_{X}\right|^{2}<\infty\right\} .
\end{gathered}
$$

- The fractional power of operator $A$ is defined as follows:

$$
\begin{gathered}
\underset{x \in D\left(A^{\beta}\right), \beta \in(0,1)}{\forall} A^{\beta} x=\sum_{i=1}^{\infty} \sum_{j=1}^{m_{i}} \lambda_{i}^{\beta}<x, \phi_{i j}>_{X} \phi_{i j} \\
D\left(A^{\beta}\right)=\left\{x \in X: \sum_{i=1}^{\infty} \sum_{j=1}^{m_{i}} \lambda_{i}^{2 \beta}\left|<x, \phi_{i j}>_{X}\right|^{2}<\infty\right\} .
\end{gathered}
$$

- Operator $A^{\beta}, 0<\beta<1$ is also selfadjoint and positive-definite with domain $\mathrm{D}\left(A^{\beta}\right)$ dense in $X$. 


\section{The transformation of the state equation}

Using the spectral resolution of the state operator $A$ and its properties (4)-(9) we can transform the infinite dimensional dynamical system, given by the abstract differential equation (1), into equivalent form of the infinite series of the finite dimensional second order linear dynamical systems with constant coefficients of the following form [5]:

$$
\begin{aligned}
& \frac{d^{2} x_{i}(t)}{d t^{2}}+\operatorname{diag}\left[\alpha_{i}^{*} \ldots \alpha_{i}^{*}\right] \frac{d x_{i}(t)}{d t}+\operatorname{diag}\left[\beta_{i}^{*} \ldots \beta_{i}^{*}\right] x_{i}(t) \\
& =\sum_{k=0}^{M} B_{i k}^{*} u\left(t-h_{k}\right), \quad i=1,2,3, \ldots
\end{aligned}
$$

where $B_{i k}^{*}$ is the following matrix [5]:

$$
B_{i k}^{*}=\left[\begin{array}{ccccc}
<b_{k 1}, \phi_{i 1}>_{X} & \ldots & <b_{k l_{2}}, \phi_{i 1}>_{X} & \ldots & <b_{k p}, \phi_{i 1}>_{X} \\
\vdots & \ddots & \vdots & \ddots & \vdots \\
<b_{k 1}, \phi_{i l_{1}}>_{X} & \ldots & <b_{k l_{2}}, \phi_{i l_{1}}>_{X} & \ldots & <b_{k p}, \phi_{i l_{1}}>_{X} \\
\vdots & \ddots & \vdots & \ddots & \vdots \\
<b_{k 1}, \phi_{i m_{i}}>_{X} & \ldots & <b_{k l_{2}}, \phi_{i m_{i}}>_{X} & \ldots & <b_{k p}, \phi_{i m_{i}}>_{X}
\end{array}\right] \quad i=1,2,3, \ldots
$$

and $x_{i}(t)$ is a vector given by the equality (12):

$$
x_{i}(t)=\left[\begin{array}{lllll}
x_{i 1}(t) & \cdots & x_{i l}(t) & \cdots & x_{i m_{i}}(t)
\end{array}\right]^{T},
$$

where $x_{i j}(t)$ denotes the $(i j)^{\text {th }}$ coefficient of the Fourier series of spectral representation for the element $x$ in the state space $X$. The coefficients are explicitly given by the inner product between element in the state space $X$ and the appropriate eigenfunctions $\phi_{i j}$ of the operator $\mathrm{A}$ :

$$
x_{i j}(t)=<x(t), \phi_{i j}>_{X} \quad i=1,2,3, \ldots \quad j=1,2, \ldots, m_{i}
$$

Additionally, in the series of the equations (10) there exist constant coefficients $\alpha_{i}^{*}$ and $\beta_{i}^{*}$, which are defined by the equalities (14) and (15) [5] pp.295:

$$
\begin{gathered}
\alpha_{i}^{*}=2\left(\alpha_{0}+\alpha_{1} \lambda_{i}+\alpha_{2} \sqrt{\lambda_{i}}\right) i=1,2,3, \ldots \\
\beta_{i}^{*}=\beta_{0}+\beta_{1} \lambda_{i}+\beta_{2} \sqrt{\lambda_{i}} \quad i=1,2,3, \ldots
\end{gathered}
$$

Basing on the infinite series of the equations (10) we can transform given system (1) to the more convenient form in the control theory, namely the form of infinite series of the set of first order finite-dimensional ordinary finite dimensional differential equations with constant coefficients (16) as follows [5]: 
Pobrane z czasopisma Annales AI- Informatica http://ai.annales.umcs.pl

$$
\dot{\zeta}_{i}(t)=A_{i} \varsigma_{i}(t)+\sum_{k=0}^{M} B_{i k} u\left(t-h_{k}\right), \quad i=1,2,3, \ldots
$$

where the state vector is given by the equality (17) [5]:

$\varsigma_{i}(t)=\left[\begin{array}{llllllll}\xi_{i 1}(t) & \mu_{i 1}(t) & \cdots & \xi_{i l}(t) & \mu_{i l}(t) & \cdots & \xi_{i m_{i}}(t) & \mu_{i m_{i}}(t)\end{array}\right]^{T}, i=1,2,3, \ldots$

and the state matrix $A_{i}$ and the input matrix $B_{i}$ have form (18), (19) respectively [5]:

$$
\begin{aligned}
& A_{i}=\left[\begin{array}{cccccccc}
0 & 1 & \cdots & 0 & 0 & \cdots & 0 & 0 \\
-\beta_{i}^{*} & -\alpha_{i}^{*} & \cdots & 0 & 0 & \cdots & 0 & 0 \\
\vdots & \vdots & \ddots & \vdots & \vdots & \ddots & \vdots & \vdots \\
0 & 0 & \cdots & 0 & 1 & \cdots & 0 & 0 \\
0 & 0 & \cdots & -\beta_{i}^{*} & -\alpha_{i}^{*} & \cdots & 0 & 0 \\
\vdots & \vdots & \ddots & \vdots & \vdots & \ddots & \vdots & \vdots \\
0 & 0 & \cdots & 0 & 0 & \cdots & 0 & 1 \\
0 & 0 & \cdots & 0 & 0 & \cdots & -\beta_{i}^{*} & -\alpha_{i}^{*}
\end{array}\right] i=1,2,3, \ldots \\
& B_{i k}=\left[\begin{array}{ccccc}
0 & \cdots & 0 & \cdots & 0 \\
\left\langle b_{k 1}, \phi_{i 1}\right\rangle_{X} & \cdots & \left\langle b_{k l_{2}}, \phi_{i 1}\right\rangle_{X} & \cdots & \left\langle b_{k p}, \phi_{i 1}\right\rangle_{X} \\
\vdots & \ddots & \vdots & \ddots & \vdots \\
0 & \cdots & 0 & \cdots & 0 \\
\left\langle b_{k 1}, \phi_{i l_{1}}\right\rangle_{X} & \cdots & \left\langle b_{k l_{2}}, \phi_{i l_{1}}\right\rangle_{X} & \cdots & \left\langle b_{k p}, \phi_{i l_{1}}\right\rangle_{X} \\
\vdots & \ddots & \vdots & \ddots & \vdots \\
0 & \cdots & 0 & \cdots & 0 \\
\left\langle b_{k 1}, \phi_{i m_{i}}\right\rangle_{X} & \cdots & \left\langle b_{k l_{2}}, \phi_{i m_{i}}\right\rangle_{X} & \cdots & \left\langle b_{k p}, \phi_{i m_{i}}\right\rangle_{X}
\end{array}\right]=\left[b_{k l_{1} l_{2}}^{(i)}\right]_{2 m_{i} \times p} i=1,2,3, \ldots
\end{aligned}
$$

The variables $\xi_{i j}(t), \mu_{i j}(t)$ are defined by formula (20) as follows [5]:

$$
\left\{\begin{array}{l}
\xi_{i j}(t)=x_{i j}(t) \\
\mu_{i j}(t)=\dot{x}_{i j}(t)
\end{array}, \quad i=1,2,3, \ldots \quad j=1,2, \ldots, m_{i} .\right.
$$

\section{The Jordan decomposition of the state matrix}

The Jordan decomposition of the state matrix (18) is investigated in paper [5] and now let us recall the results. This matrix has two distinct eigenvalues $s_{i 1}, s_{i 2}$ each with the same multiplicity $m_{i}$ [5] pp.297:

$$
s_{i 1}=\frac{-\alpha_{i}^{*}-\sqrt{\alpha_{i}^{* 2}-4 \beta_{i}^{*}}}{2}, \quad s_{i 2}=\frac{-\alpha_{i}^{*}+\sqrt{\alpha_{i}^{* 2}-4 \beta_{i}^{*}}}{2} \quad i=1,2,3, \ldots
$$


Depending on the particular values of the coefficients $\alpha_{i} \geq 0, \beta_{i} \geq 0, i=0,1,2$ the Jordan's canonical forms $J\left(A_{i}\right)$ and the transition matrices $T\left(A_{i}\right)$ have the following forms, respectively [5]:

3.1. Case 1: $\beta_{i}^{*} \neq 0, \beta_{i}^{*} \neq \frac{\alpha_{i}^{* 2}}{4}$

$$
\begin{aligned}
& J\left(A_{i}\right)=\operatorname{diag}[\underbrace{s_{i 1} \ldots s_{i 1}}_{m_{i}-\text { times }} \underbrace{s_{i 2} \ldots s_{i 2}}_{m_{i}-\text { times }}] \quad i=1,2,3, \ldots,
\end{aligned}
$$

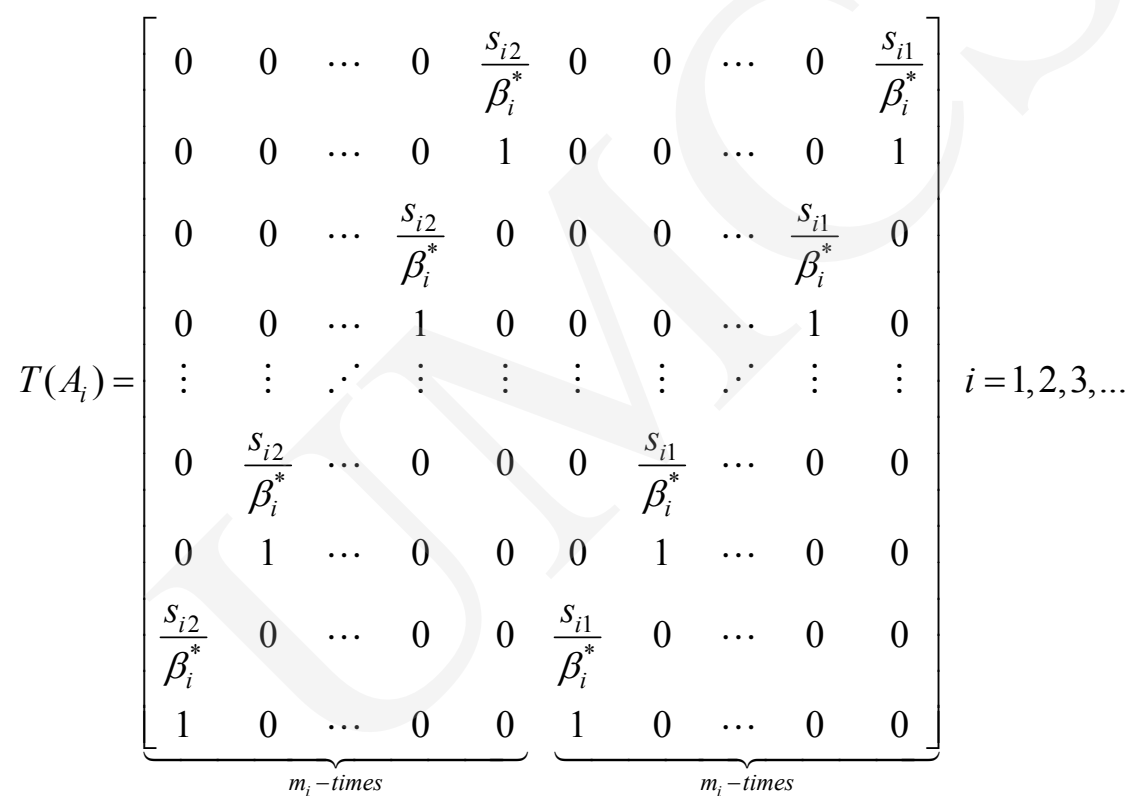

3.2. Case 2: $\beta_{i}^{*}=0, \alpha_{i}^{*} \neq 0$

$$
J\left(A_{i}\right)=\operatorname{diag} \underbrace{[0 \ldots 0}_{m_{i} \text {-times }} \underbrace{-\alpha_{i}^{*} \ldots-\alpha_{i}^{*}}_{m_{i} \text {-times }}] \quad i=1,2,3, \ldots,
$$


Pobrane z czasopisma Annales AI- Informatica http://ai.annales.umcs.pl

Data: 26/04/2023 16:35:53

$$
T\left(A_{i}\right)=\left[\begin{array}{cccccccccc}
0 & 0 & \cdots & 0 & 1 & 0 & 0 & \cdots & 0 & -\frac{1}{\alpha_{i}^{*}} \\
0 & 0 & \cdots & 0 & 0 & 0 & 0 & \cdots & 0 & 1 \\
0 & 0 & \cdots & 1 & 0 & 0 & 0 & \cdots & -\frac{1}{\alpha_{i}^{*}} & 0 \\
0 & 0 & \cdots & 0 & 0 & 0 & 0 & \cdots & 1 & 0 \\
\vdots & \vdots & . & \vdots & \vdots & \vdots & \vdots & \cdots & \vdots & \vdots \\
0 & 1 & \cdots & 0 & 0 & 0 & -\frac{1}{\alpha_{i}^{*}} & \cdots & 0 & 0 \\
0 & 0 & \cdots & 0 & 0 & 0 & 1 & \cdots & 0 & 0 \\
1 & 0 & \cdots & 0 & 0 & -\frac{1}{\alpha_{i}^{*}} & 0 & \cdots & 0 & 0 \\
0 & 0 & \cdots & 0 & 0 & 1 & 0 & \cdots & 0 & 0
\end{array}\right] i=1,2,3, \cdots
$$

3.3. Case 3: $\beta_{i}^{*}=\frac{\alpha_{i}^{* 2}}{4}, \alpha_{i}^{*} \neq 0$

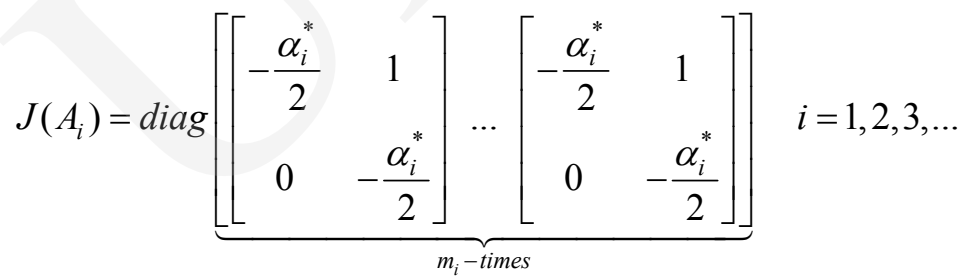

$$
\begin{aligned}
& T\left(A_{i}\right)=\underbrace{\left[\begin{array}{ccccc}
0 & 0 & \cdots & -\frac{2}{\alpha_{i}^{*}} & -\frac{4}{\alpha_{i}^{* 2}} \\
0 & 0 & \cdots & 1 & 0 \\
\vdots & \vdots & \ddots & \vdots & \vdots \\
-\frac{2}{\alpha_{i}^{*}} & -\frac{4}{\alpha_{i}^{* 2}} & \cdots & 0 & 0 \\
1 & 0 & \cdots & 0 & 0
\end{array}\right]}_{2 m_{i} \text {-times }} i=1,2,3, \ldots
\end{aligned}
$$

3.4. Case 4: $\alpha_{i}^{*}=\beta_{i}^{*}=0$ 


$$
J\left(A_{i}\right)=\operatorname{diag} \underbrace{\left.\left[\begin{array}{ll}
0 & 1 \\
0 & 0
\end{array}\right] \cdots\left[\begin{array}{ll}
0 & 1 \\
0 & 0
\end{array}\right]\right]}_{m_{i} \text {-times }} i=1,2,3, \ldots
$$

$$
T\left(A_{i}\right)=\underbrace{\left[\begin{array}{ccccc}
0 & 0 & \cdots & 1 & 0 \\
0 & 0 & \cdots & 0 & 1 \\
\vdots & \vdots & \ddots & \vdots & \vdots \\
1 & 0 & \cdots & 0 & 0 \\
0 & 1 & \cdots & 0 & 0
\end{array}\right]}_{2 m_{i} \text {-times }} i=1,2,3, \ldots
$$

Obviously the following identity holds true for all the Jordan's decomposition cases:

$$
A_{i}=T\left(A_{i}\right) \cdot J\left(A_{i}\right) \cdot T^{-1}\left(A_{i}\right) \quad i=1,2,3, \ldots
$$

Now let us verify whether the operator $A_{i}(18)$ is the infinitesimal generator of an analytic semigroup. Following this aim let us calculate some auxiliary limits. By the assumptions $\alpha_{i} \geq 0, \beta_{i} \geq 0, i=0,1,2$, formulas (14), (15) and the property of the state operator (4) it can be stated that ((35), (36)):

$$
\begin{gathered}
\lim _{i \rightarrow \infty} \operatorname{Re}\left[\alpha_{i}^{*}+\sqrt{\alpha_{i}^{* 2}-4 \beta_{i}^{*}}\right] \neq-\infty \\
\lim _{i \rightarrow \infty} \operatorname{Re}\left[\alpha_{i}^{*}+\sqrt{\alpha_{i}^{* 2}-4 \beta_{i}^{*}}\right]=\lim _{i \rightarrow \infty} \operatorname{Re}\left[\frac{4 \beta_{i}^{*}}{\alpha_{i}^{*}+\sqrt{\alpha_{i}^{* 2}-4 \beta_{i}^{*}}}\right] \neq-\infty .
\end{gathered}
$$

Moreover, from these assumptions and (14), (15) we have (37):

$$
\underset{i=1,2,3, \ldots}{\forall} \alpha_{i}^{*} \geq 0, \beta_{i}^{*} \geq 0, \operatorname{Re}\left[\alpha_{i}^{*}+\sqrt{\alpha_{i}^{* 2}-4 \beta_{i}^{*}}\right] \geq 0 .
$$

Now based on (35)-(37) we can calculate (38), (39):

$$
\begin{aligned}
& \lim _{i \rightarrow \infty} \operatorname{Re}\left[s_{i 1}\right]=\lim _{i \rightarrow \infty} \operatorname{Re} \sqrt{\frac{-\alpha_{i}^{*}-\sqrt{\alpha_{i}^{* 2}-4 \beta_{i}^{*}}}{2}}= \\
& =\operatorname{Re} \sqrt{\frac{-\lim _{i \rightarrow \infty}\left(\alpha_{i}^{*}+\sqrt{\alpha_{i}^{* 2}-4 \beta_{i}^{*}}\right)}{2}<\infty} \\
& \lim _{i \rightarrow \infty} \operatorname{Re}\left[s_{i 2}\right]=\lim _{i \rightarrow \infty} \operatorname{Re} \sqrt{\frac{-\alpha_{i}^{*}+\sqrt{\alpha_{i}^{* 2}-4 \beta_{i}^{*}}}{2}}= \\
& =\operatorname{Re} \sqrt{\frac{-\lim _{i \rightarrow \infty}\left(\alpha_{i}^{*}-\sqrt{\alpha_{i}^{* 2}-4 \beta_{i}^{*}}\right)}{2}}<\infty
\end{aligned}
$$


so the operator $A_{i}(18)$ is the infinitesimal generator of an analytic semigroup.

\section{Basic notions}

Following the aim of analyzing the approximate controllability of infinite dimensional system with delays (1) at first let us present this notion in the case of finite dimensional systems. At first let us consider the linear stationary dynamical system, described by the differential equation without delays in control [6] pp.5:

$$
\dot{x}(t)=A_{0} x(t)+B_{0} u(t), \quad t \geq 0
$$

\subsection{Definition $4.1[6]$}

The dynamical system (40) is said to be controllable, if and only if there exists such a control $u(t)$, which will transfer the system from any given initial state to any final state in the control space in the finite time.

\subsection{Theorem 4.2 [6] pp.16, [7] pp.70}

The dynamical system (40) is controllable if and only if condition (41) holds true:

$$
\operatorname{rank}\left[B_{0}\left|A_{0} B_{0}\right| A_{0}^{2} B_{0}|\ldots| A_{0}^{n-1} B_{0}\right]=n
$$

Now let us consider linear stationary dynamical system, described by the differential equation with delays in control (42) [6] pp.196:

$$
\dot{x}(t)=A_{0} x(t)+\sum_{k=0}^{M} B_{0 k} u\left(t-h_{k}\right), \quad t \geq 0
$$

where $A_{0}, B_{0}$ are the constant matrices with dimensions respectively $\mathrm{n} \times \mathrm{n}, \mathrm{n} \times \mathrm{p}$.

For the dynamical system of form (42) besides the instantaneous state $x(t) \in R^{n}$, we introduce also the notion of the so-called complete state at time $t, z(t)=\left\{x(t), u_{t}(s)\right\}$, where $u_{t}(s)=u(s)$ for $s \in\left[t-h_{M}, t\right][15]$. Therefore we distinguish two basic notions of controllability for dynamical systems (42), namely: relative controllability and absolute controllability [6] pp.195. Definitions 4.3 and 4.4 taken from position [6] pp.195 are adapted to the dynamical system (42) i.e. with multiple, lumped time-invariant delays in control.

\subsection{Definition 4.3 [6] pp.195}

Dynamical system (42) is said to be relatively controllable in $\left[t_{0}, t_{1}\right]$, if for any initial complete state $z\left(t_{0}\right)$ and any vector $x_{1} \in R^{n}$, there exists a control 
$u \in L^{2}\left(\left[t_{0}, t_{1}\right], R^{p}\right)$ such that the corresponding trajectory $x\left(t, z\left(t_{0}\right), u\right)$ of dynamical system (42) satisfies the following condition (43):

$$
x\left(t_{1}, z\left(t_{0}\right), u\right)=x_{1} .
$$

\subsection{Definition 4.4 [6] pp.195}

Dynamical system (42) is said to be absolutely controllable in $\left[t_{0}, t_{1}\right]$, if for any initial complete state $z\left(t_{0}\right)$, any vector $x_{1} \in R^{n}$ and an arbitrary function $w \in L^{2}\left(\left[0, h_{M}\right], R^{p}\right)$ there exists a control $u \in L^{2}\left(\left[t_{0}, h_{M}\right], R^{p}\right)$ such that the complete state at time $t_{1}$ of dynamical system (42) satisfies the following condition (44):

$$
z\left(t_{1}\right)=\left\{x_{1}, w\right\} .
$$

There are some known theorems for verifying the relative and absolute controllability of linear time varying systems with delays and control. Let us present two main theorems adapted to the stationary dynamical system of the form (42).

\subsection{Theorem $4.5[6]$ pp.202}

Dynamical system (42) is relatively controllable in $\left[t_{0}, t_{1}\right]$, if and only if the dynamical system without delays in control, of the form

$$
\dot{x}(t)=A_{0} x(t)+\tilde{B}_{0} w(t), \quad t \in\left[t_{0}, t_{1}\right]
$$

where

$$
\tilde{B}=\left[B_{00}\left|B_{01}\right| \ldots \mid B_{0(k-1)}\right], \quad t \in\left[t_{0}, t_{1}\right], w \in R^{k p}
$$

is controllable in $\left[t_{0}+h_{k-1}, t_{1}\right]$.

\subsection{Theorem $4.6[6]$ pp.207}

Dynamical system (42) is absolutely controllable in $\left[t_{0}, t_{1}\right]$ if and only if the dynamical system without delays in control, of the form

$$
\dot{x}(t)=A_{0} x(t)+\hat{B}_{0} u(t), \quad t \in\left[t_{0}, t_{1}\right],
$$

where

$$
\hat{B}_{0}=\sum_{k=0}^{M} e^{-A_{0} h_{k}} B_{k 0}
$$

is controllable in $\left[t_{0}, t_{1}-h_{M}\right]$. 


\section{Approximate controllability analysis}

Both theorems 4.5 and 4.6 base on the transformation of the system with delays in controls into the corresponding system without delays in controls. It can be noticed that that the state matrix in the corresponding systems (45) and (47) remains the same as in the investigated system with delays in controls (42). Let us return to the considered in this paper second order infinite dimensional dynamical system (1) in the form of the series (16). We will analyze its approximate relative and approximate absolute controllability by theorems 4.5 and 4.6, so the system series (16) in both the corresponding forms (45) and (47) has the same state matrices $A_{i}$ like in the system (16). In point 3 we recalled the Jordan decomposition of the state matrices $A_{i}$. This form is very convenient for testing the controllability of a given dynamical system-involves only calculating the $T_{i}^{-1} B_{i k}$ term, instead of calculating the $n-1$ terms of the form $A_{i}^{l} B_{i}$ in the block matrix (41) necessary in case of using theorem 4.2. The general conditions of the controllability for the stationary, linear, finite dimensional without delays in the control dynamical system in the Jordan canonical form have been formulated by C.T.Chen in Chapter 5.5 of work [8] and have been recalled in paper [6] by theorem 1.5.1. In the next subchapters we use theorems 4.5 and 4.6 and the Chen's theorem to find the conditions of approximate absolute and approximate relative controllability of a given system (1).

\subsection{Theorem 5.1}

Dynamical system (1) is approximately absolute controllable at any time if and only if the infinite series of equalities (49) is fulfilled:

$$
\left\{\begin{array}{l}
\operatorname{rank} \sum_{k=0}^{M} e^{-s_{i} h_{k}} B_{i k}^{*}=m_{i} \\
\operatorname{rank} \sum_{k=0}^{M} e^{-s_{i 2} h_{k}} B_{i k}^{*}=m_{i}
\end{array} \quad i=1,2,3, \ldots\right.
$$

where $B_{i k}^{*}$ is given by (11).

Proof of the absolute controllability

The proof will be given as an example for case 1 of the Jordan decomposition (paragraph 3.1). We will prove the conditions of the absolute controllability of system (1) in the form of series (16) by the theorem 4.6 and mentioned Chen's theorem [6] pp. 25. At first let us calculate the matrix $\hat{B}$ for the system (16):

$$
\hat{B}_{i}=\sum_{k=0}^{M} e^{-A_{i} h_{k}} B_{i k}=\sum_{k=0}^{M} T_{i} e^{-J_{i} h_{k}} T_{i}^{-1} B_{i k}, \quad i=1,2,3, \ldots
$$


The key role in the Chen's theorem plays the $T_{i}^{-1} \hat{B}_{i}$ term. The inverse matrix $T_{i}^{-1}$ is calculated in work [5] pp. 300, so from (50) we have:

$$
\begin{aligned}
& T_{i}^{-1} \hat{B}_{i}=\sum_{k=0}^{M} e^{-J_{i} h_{k}} T_{i}^{-1} B_{i k}=
\end{aligned}
$$

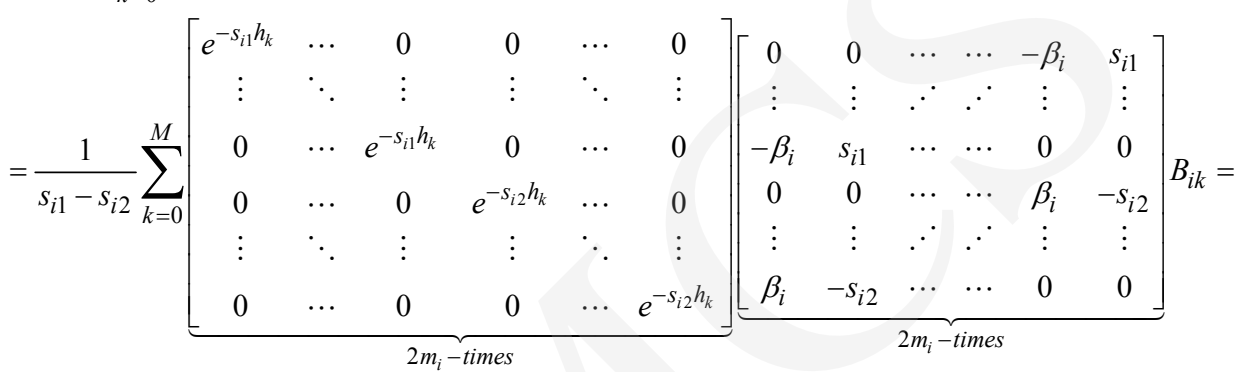

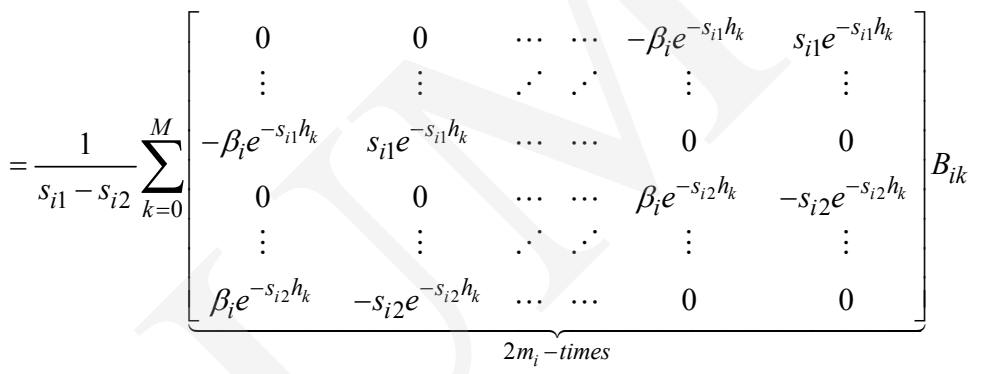

Considering that the odd rows in the series of the matrices $B_{i k}$ (19) are zero from (51) we have directly (52):

$$
T_{i}^{-1} \hat{B}_{i}=\frac{1}{s_{i 1}-s_{i 2}} \sum_{k=0}^{M}\left[\begin{array}{ccc}
s_{i 1} e^{-s_{i 1} h_{k}} b_{k n 1}^{(i)} & \cdots & s_{i 1} e^{-s_{i 1} h_{k}} b_{k n p}^{(i)} \\
\vdots & \ddots & \vdots \\
s_{i 1} e^{-s_{i} h_{k}} b_{k 21}^{(i)} & \cdots & s_{i 1} e^{-s_{i 1} h_{k}} b_{k 2 p}^{(i)} \\
-s_{i 2} e^{-s_{i 2} h_{k}} b_{k n 1}^{(i)} & \cdots & -s_{i 2} e^{-s_{i 2} h_{k}} b_{k n p}^{(i)} \\
\vdots & \ddots & \vdots \\
-s_{i 2} e^{-s_{i 2} h_{k}} b_{k 21}^{(i)} & \cdots & -s_{i 2} e^{-s_{i 2} h_{k}} b_{k 2 p}^{(i)}
\end{array}\right]
$$

Now let us return to the verification of the controllability of dynamical system (1) in the form (16). Applying the Chen's theorem to it, based on formula (52), considered system (16) presented in the corresponding form without delays (47) with the input matrix $\hat{B}_{i}$ (given by (50)) is approximately controllable if and only if series (53), (54) are fulfilled: 


$$
\begin{aligned}
& \operatorname{rank}\left[\frac{1}{s_{i 1}-s_{i 2}} \sum_{k=0}^{M}\left[\begin{array}{ccc}
s_{i 1} e^{-s_{i 1} h_{k}} b_{k n 1}^{(i)} & \cdots & s_{i 1} e^{-s_{i 1} h_{k}} b_{k n p}^{(i)} \\
\vdots & \ddots & \vdots \\
s_{i 1} e^{-s_{i 1} h_{k}} b_{k 21}^{(i)} & \cdots & s_{i 1} e^{-s_{i 1} h_{k}} b_{k 2 p}^{(i)}
\end{array}\right]\right]=m_{i}, \quad i=1,2,3, \ldots \\
& \operatorname{rank}\left[\frac{1}{s_{i 1}-s_{i 2}} \sum_{k=0}^{M}\left[\begin{array}{ccc}
s_{i 2} e^{-s_{i 2} h_{k}} b_{k n 1}^{(i)} & \cdots & s_{i 2} e^{-s_{i 2} h_{k}} b_{k n p}^{(i)} \\
\vdots & \ddots & \vdots \\
s_{i 2} e^{-s_{i 2} h_{k}} b_{k 21}^{(i)} & \cdots & s_{i 2} e^{-s_{i 2} h_{k}} b_{k 2 p}^{(i)}
\end{array}\right]\right]=m_{i}, \quad i=1,2,3, \ldots
\end{aligned}
$$

Division of matrix (52) into two series of rank conditions (53), (54) follows from the mentioned Chen's theorem and the Jordan decomposition of the state matrices $A_{i}$ (18) in case 1 (point 3.1, equation (23)): we have two Jordan blocks corresponding to two different eigenvalues $s_{i 1}, s_{i 2}$ (21). By the basic linear algebra rules from (53) we have (55):

$$
\operatorname{rank} \sum_{k=0}^{M} s_{i 1} e^{-s_{i l} h_{k}}\left(B_{i k}^{*}\right)^{\prime}=m_{i}, \quad i=1,2,3, \ldots,
$$

where $\left(B_{i k}^{*}\right)^{\prime}$ matrices can be obtained from the input matrices $B_{i k}^{*}(11)$ by reversing the order of the rows. By this notice from (53)-(55) we directly have the condition from the thesis of proving theorem 5.1 (49).

The Chen's theorem gives the conditions for controllability of time-invariant system of the Jordan form, but in theorems 4.5 and 4.6 we have controllability in closed time ranges. But by Lemma 1.4.1 from the work [6] pp. 16 for the timeinvariant systems the notions of the controllability in a closed time range and the controllability at any time are equivalent.

The pProof in the remaining cases 2-4 goes similarly and detailed calculations will be omitted. A special attention should be paid to cases 3 and 4 because they correspond to two-dimensional Jordan blocks with ones above the main diagonal in the Jordan decomposition of the state matrices, presented in points 3.3 and 3.4 (formulas (29) and (32)).

Q.E.D

\subsection{Theorem 5.2}

Dynamical system (1) is approximately relatively controllable if and only if the infinite series of equalities (56) are fulfilled:

$$
\operatorname{rank}\left[B_{i 0}^{*}\right]=m_{i}, \quad i=1,2,3, \ldots,
$$

where $B_{i 0}^{*}$ is given by (11). 


\section{Proof of the relative controllability}

The proof will also be given for an example for case 1 of the Jordan decomposition (paragraph 3.1). The proof bases on theorem 4.5 and the Chen's theorem [6] pp. 25. The $T_{i}^{-1} \tilde{B}_{i}$ term can be easily calculated (57):

$$
T_{i}^{-1} \tilde{B}_{i}=T_{i}^{-1}\left[B_{i 0}\right]=\frac{1}{s_{i 1}-s_{i 2}} \cdot \frac{s_{i 1}\left[\left(B_{i 0}^{*}\right)^{\prime}\right]}{-s_{i 2}\left[\left(B_{i 0}^{*}\right)^{\prime}\right]} \quad i=1,2,3, \ldots,
$$

where $\left(B_{i k}^{*}\right)^{\prime}$ matrices can be obtained from the input matrices $B_{i k}^{*}(11)$ by reversing the order of the rows, and the matrix $B_{i 0}$ is given by (19). From (57) and the mentioned Chen's theorem there directly follows the series of equalities (56) for the approximate relative controllability in the range $\left[t_{0}, t_{1}\right]$. Also here by Lemma 1.4.1 from work [6] pp. 16 for the time-invariant systems the notions of the controllability in a closed time range and the controllability at any time are equivalent. The proof in the remaining cases 2-4 also proceed similarly. Notice from the proof of the absolute controllability pertaining to cases 3 and 4 holds true in the relative controllability.

Q.E.D.

\section{Mechanical example}

Let us consider a mechanical system described by the following linear partial differential equation:

$$
\begin{aligned}
& \frac{\partial^{2} x(z, t)}{\partial t^{2}}+\frac{\partial^{4} x(z, t)}{\partial z^{4}}+2 \frac{\partial^{5} x(z, t)}{\partial z^{4} \partial t}-6 \frac{\partial^{3} x(z, t)}{\partial z^{2} \partial t}-3 \frac{\partial^{2} x(z, t)}{\partial z^{2}}= \\
& =z u_{1}(t)+z^{3} u_{1}(t-1)+2 u_{2}(t)+u_{2}(t-1)
\end{aligned}
$$

with initial conditions (59), (60):

$$
\begin{aligned}
& x(z, 0)=x_{0}(z), \quad z \in\left(0, L_{0}\right), \\
& \frac{\partial x(z, 0)}{\partial t}=x_{1}(z), \quad z \in\left(0, L_{0}\right)
\end{aligned}
$$

and boundary conditions:

$$
x(0, t)=x\left(L_{0}, t\right)=\frac{\partial^{2} x(0, t)}{\partial z^{2}}=\frac{\partial^{2} x\left(L_{0}, t\right)}{\partial z^{2}}=0, \quad t>0
$$

The function $x(z, t)$ is equal to the movement of the considered elastic beam in the $\mathrm{Y}$ axis direction in the time moment $t>0$ and in the point $z\left(0<z<L_{0}\right)$. The first two terms in equation (58) are the only terms taken into account for the ideally springy elastic beam. The next two terms are modelling the phenomenon of the internal friction and the remaining fifth term represents the effect of axial 
force on the beam. The boundary conditions correspond to hinged ends of the beam. More detailed description of these terms and the phenomenon they are describe can be found in papers $[4,9,10,11]$.

\subsection{The definition of the state differential operator}

Let us define the linear unbounded differential operator $A: D(A) \subset H \rightarrow H$ $[9,11]$ in the following way:

$$
\begin{gathered}
A x(z)=\frac{\partial^{4} x(z)}{\partial z^{4}}, x \in D(A) \\
D(A)=\left\{x(z) \in H_{0}^{4,2}\left(\left[0, L_{0}\right], R\right): \frac{d^{4}}{d z^{4}} x(z) \in L^{2}\left(\left[0, L_{0}\right], R\right), \quad x(0)=x\left(L_{0}\right)=\frac{d^{2} x}{d z^{2}}(0)=\frac{d^{2} x}{d z^{2}}\left(L_{0}\right)=0\right\}
\end{gathered}
$$

where $H_{0}^{4,2}\left(\left[0, L_{0}\right], R\right)$ denotes the fourth order Sobolev space defined in the range $\left[0, L_{0}\right]$.

It can be proved $[9,11]$ that the eigenvalues $\lambda_{i}$ and the eigenfunctions $\phi_{i}(z)$ of the operator $A$ have the form (64), (65):

$$
\begin{gathered}
\lambda_{i}=\left(\frac{i \pi}{L_{0}}\right)^{4} \quad i=1,2,3, \ldots \\
\phi_{i}(z)=\sqrt{\frac{2}{L_{0}}} \sin \frac{\pi i z}{L_{0}} \quad i=1,2,3, \ldots
\end{gathered}
$$

and the operator $A$ is linear, self-adjoint and positively defined. Particularly it can be defined by the following fractional power (8) of the operator $A$ [9]:

$$
\begin{gathered}
A^{\frac{1}{2}} x=-\frac{\partial^{2} x}{\partial z^{2}} \\
D\left(A^{\frac{1}{2}}\right)=\left\{x \in H_{0}^{2,2}\left(\left[0, L_{0}\right], R\right): \frac{d^{2}}{d z^{2}} x(z) \in L^{2}\left(\left[0, L_{0}\right], R\right): x(0)=x\left(L_{0}\right)=0\right\}
\end{gathered}
$$

where $H_{0}^{2,2}$ denotes the second order Sobolev space on the interval $\left[0, L_{0}\right]$ and $D(A) \subset D\left(A^{1 / 2}\right)$.

\subsection{The state equation}

Applying operator $A$ (62) to partial differential equation (58) with boundary conditions (61) we obtain the following abstract, ordinary second order differential equation with respect to $t$ in the Sobolev space $H$ : 


$$
\frac{d^{2} x(t)}{d t^{2}}+2\left(A+3 A^{\frac{1}{2}}\right) \frac{d x(t)}{d t}+\left(A+3 A^{\frac{1}{2}}\right) x(t)=\sum_{k=0}^{1} B_{k} u\left(t-h_{k}\right), \quad t>0,(68)
$$

where:

$$
\begin{gathered}
\frac{d^{2} x(t)}{d t^{2}}, \frac{d x(t)}{d t}, x(t) \in H, \\
B_{0}=\left[\begin{array}{ll}
z & 2
\end{array}\right] \quad B_{1}=\left[\begin{array}{ll}
z^{3} & 1
\end{array}\right] \\
h_{0}=0, h_{1}=1
\end{gathered}
$$

It is easy to see that equation (68) has form of the dynamical system (1) after introduction of the following coefficients:

$$
\alpha_{0}=0, \quad \alpha_{1}=1, \quad \alpha_{2}=3, \quad \beta_{0}=0, \quad \beta_{1}=1, \quad \beta_{2}=3
$$

\subsection{The approximate absolute controllability analysis of the infinite dimensional mechanical system}

In this subchapter the analysis of the approximate absolute controllability of given infinite dimensional dynamical system (58) will be performed. Also this dynamical system will be represented by the infinite series of the finite dimensional dynamical systems (16). These aims will be accomplished theorem 5.1. First, let us calculate the coefficients $\alpha_{i}^{*}$ and $\beta_{i}^{*}$ on the basis of its definitions (14), (15):

$$
\begin{aligned}
& \alpha_{i}^{*}=2\left(\frac{i \pi}{L_{0}}\right)^{4}+6\left(\frac{i \pi}{L_{0}}\right)^{2}, \quad i=1,2,3, \ldots, \\
& \beta_{i}^{*}=\left(\frac{i \pi}{L_{0}}\right)^{4}+3\left(\frac{i \pi}{L_{0}}\right)^{2} \quad i=1,2,3, \ldots .
\end{aligned}
$$

In theorem 5.1 there are needed the matrices $B_{i k}^{*}$. Considering (65), (70) we have (75), (76):

$$
\begin{aligned}
& B_{i 0}^{*}=\sqrt{\frac{2}{L_{0}}}\left[\int_{0}^{L_{0}} z \sin \frac{\pi i z}{L_{0}} d z \quad 2 \int_{0}^{L_{0}} \sin \frac{\pi i z}{L_{0}} d z\right], \quad i=1,2,3, \ldots \\
& B_{i 1}^{*}=\sqrt{\frac{2}{L_{0}}}\left[\int_{0}^{L_{0}} z^{3} \sin \frac{\pi i z}{L_{0}} d z \quad \int_{0}^{L_{0}} \sin \frac{\pi i z}{L_{0}} d z\right], \quad i=1,2,3, \ldots
\end{aligned}
$$

Let us look at the thesis of theorem 5.1. In the case of the operator (62), (63) $m_{i}=1$, so the rank in condition (49) is being testified on the 2-element horizontal vector, and the conditions are fulfilled if and only if any of the vector elements is 
nonzero. Moreover, the $e^{-s_{i 1} h_{k}}, e^{-s_{i 2} h_{k}}$ coefficients are nonzero. Now let us look at the matrices $B_{i k}^{*}(75),(76)$. By the Lemma 1 from work [5] pp. 312-313, the integrals $\int_{0}^{L_{0}} z \sin \frac{\pi i z}{L_{0}} d z, \int_{0}^{L_{0}} z^{3} \sin \frac{\pi i z}{L_{0}} d z$ are positive. It's also easy to check that the integral $\int_{0}^{L_{0}} \sin \frac{\pi i z}{L_{0}} d z$ is nonnegative. Thus in the sums $\sum_{k=0}^{1} e^{-s_{i 1} h_{k}} B_{i k}^{*}, \sum_{k=0}^{1} e^{-S_{i 2} h_{k}} B_{i k}^{*}$ the non-negative and non-zero $B_{i k}^{*}$ matrices are taken into the linear combination with positive exponential coefficients obviously giving non-zero vectors, so condition (49) is fulfilled.

\subsection{The approximate relative controllability analysis of the infinite dimensional mechanical system}

Theorem 5.2 by (75) and the remarks from point 6.3 are fulfilled.

\subsection{Summary of the mechanical example}

The mechanical system (58) with conditions (59)-(61) is both aproximately relative and absolute controllable at any time.

\section{Conclusions}

In the article we obtained general conditions of different types of controllability for the infinite dimensional systems. It was possible thanks to making the use of the Chen's theorem. The obtained theorems of the approximate controllability without constraints, with the cone type constraints, and with delays in control hold true for the second order of the verified infinite dimensional dynamical system. This is innovative outcome in the controllability theory field.

Moreover, it should be pointed out that the presented methods can be easily adapted to the analysis of other dynamical properties of the considered nth order system, i.e. observability, attainability, stability and optimal control.

A possible way of further investigations can be the generalisation of the presented results into the case of arbitrary eigenvalues multiplicities of the state operator. 
Pobrane z czasopisma Annales AI- Informatica http://ai.annales.umcs.pl

Data: 26/04/2023 16:35:53

Controllability of second order infinite dimensional dynamical ...

\section{References}

[1] Fattorini H.O., Some Remarks on Complete Controllability. SIAM Journal on Control and Optimization, 4 (1966) 686.

[2] Fattorini H.O., On Complete Controllability of Linear Systems. Journal of Differential Equations, 3 (1967) 391.

[3] Huang F., On The Mathematical Model with Analytic Damping. SIAM Journal on Control Optimization, 26(3) (1988) 714.

[4] Sakawa Y., Feedback Control of Second Order Evolution Equations with Damping. SIAM Journal on Control and Optimisation, 22 (1984) 343.

[5] Respondek J., Controllability of Dynamical Systems with Constraints. Systems \& Control Letters, 54/4 293.

[6] Klamka J., Controllability of Dynamical Systems. Kluwer, Dordrecht, (1991).

[7] Mitkowski W., Stabilizacja systemów dynamicznych. WNT, (1991), (in Polish).

[8] Chen C.T., Introduction to Linear System Theory. New York, Holt, Rinehart and Winston Inc.

[9] Ito K., Kunimatsu N., Stabilization of Non-Linear Distributed Parameter Vibratory System. International Journal of Control, 48 (1988) 2389.

[10] Sakawa Y., Controllability for Partial Differential Equations of Parabolic Type. SIAM Journal on Control and Optimization, 12 (1974) 389.

[11] Sakawa Y., Feedback Stabilization of Linear Diffusion System. SIAM Journal on Control and Optimization, 21(5) (1983) 667. 